\title{
Comparative Study of Radiological Changes in Hands and Feet in Patients Suffering from Early Rheumatoid Arthritis by Power Doppler Ultrasound and Direct Digital Radiography
}

\author{
Gargy Mondal ${ }^{1}$, Biswadip Ghosh ${ }^{2 *}$, Manoranjan Ghosh ${ }^{3}$, Pampa Halder ${ }^{4}$ \\ ${ }^{1}$ Department of Radiodiagnosis, Medical College, Kolkata, India; ${ }^{2}$ Department of Rheumatology, Institute of Postgraduate Medical \\ Education and Research, Kolkata, India; ${ }^{3}$ Department of Surgery, Medical College, Kolkata, India; ${ }^{4}$ Department of Ophthalmology, \\ RG Kar Medical College, Kolkata, India. \\ Email: ${ }^{\text {drbiswadip@gmail.com }}$
}

Received October 29 $9^{\text {th }}, 2013$; revised November $29^{\text {th }}$, 2013; accepted December $8^{\text {th }}, 2013$

Copyright (c) 2014 Gargy Mondal et al. This is an open access article distributed under the Creative Commons Attribution License, which permits unrestricted use, distribution, and reproduction in any medium, provided the original work is properly cited. In accordance of the Creative Commons Attribution License all Copyrights (c) 2014 are reserved for SCIRP and the owner of the intellectual property Gargy Mondal et al. All Copyright (C) 2014 are guarded by law and by SCIRP as a guardian.

\section{ABSTRACT}

Rheumatoid arthritis is a chronic multisystem disease of unknown cause. The characteristic feature of RA is persistent inflammatory synovitis. The natural history of disease is such that the early months of the disease are critical period during which reversible joint damage occurs. So early diagnosis of RA and appropriate drug application is the only way to save a patient from this crippling disease. In India, the cost of investigations is a significant factor for most of the patients. Ultrasonography or Power Doppler Ultra Sound (PDUS) has the advantage of being economic in spite of its sensitivity in assessing both inflammatory and destructive changes. The aim of the present study was to evaluate the diagnostic efficiency of PDUS in early rheumatoid arthritis. The study was performed with the patients attending Rheumatology Clinic. A total number of 106 patients of clinically suspected rheumatoid arthritis were studied as per selection criteria. Radiological examinations of hands were done by digital radiography and PDUS in a group of 53 patients, assessment of foot changes by PDUS and Digital Radiography were done in another similar group of 53 patients. Final diagnosis by ACR EULAR-2010 criteria is done for all the patients. The comparative study reveals that synovial vascularity as demonstrated by PDUS is much more effective in diagnosing early rheumatoid arthritis, both in hand and in feet than digital radiograph. PDUS of feet may yield earlier and better findings than hands, which is conventionally used in patients suffering from early rheumatoid arthritis.

\section{KEYWORDS}

R.A.-Rheumatoid Arthritis; PDUS_-Power Doppler Ultrasound; D.R._-Digital Radiography; ACR-EULAR-American College of Rheumatology (ACR) and the European League against Rheumatism (EULAR); RF—Rheumatoid Factor; CRP—c-Reactive Protein; MRI-Magnetic Resonance Imaging

\section{Introduction}

Rheumatoid Arthritis is a chronic multisystem disease of unknown cause. The potential of synovial inflammation to cause cartilage damage and bone erosions and subsequent changes in joints integrity is the hallmark of the

${ }^{*}$ Corresponding author. disease. About 1\% of the world's population is afflicted by rheumatoid arthritis, women three times more often than men.

Early diagnosis of R.A. and appropriate therapy is the only way to save a patient from this crippling disease. MRI, though very sensitive and specific for detection of early Rheumatoid arthritis, its cost is a limiting factor. The present study will help in evaluation of the role of 
digital X-ray and Power Doppler Ultrasonography for detection of early R.A.

\section{Objectives}

- Evaluation of digital radiographic Changes in early RA in both hands and feet.

- Evaluation of Power Doppler Ultrasonography (PDUS) features in early R.A. in both hands and feet.

- Comparison between changes of hands and feet in early RA.

- Correlation with ACR EULAR-2010 criteria [1].

\section{Materials and Method}

Study Area: Medical College and Hospital, Kolkata.

Study Population: Patients attending Rheumatology

Clinic of Medical College and Hospital, Kolkata, India.

Study Period: January 2011 to June 2012.

\section{Sample Design:}

1) Inclusion Criteria:

- Age more than 16 years.

- Patients having inflammatory polyarthritis of less than two years of duration with early morning stiffness greater than one hour.

2) Exclusion Criteria:

- Non inflammatory Arthritis.

- Patients with Diabetes Mellitus, Hypothyroidism.

- Patients on Steroid therapy.

Study Design: It was a Cross sectional Study. Study Tools:

- Evaluation by Digital Radiography Vander Heijde modification of sharp scoring for erosion and joint space narrowing.

Digital Radiograph was taken by PHILIPS ALLURA;

XPER FD-20 machine using appropriate Kvs and mAs [2].

- Evaluation by PDUS-Synovial Vascularity by $4 \mathrm{pt}$ scale.

Grade 0 = absence of signal, no intra-articular flow;

Grade 1 = mild, one or two vessels signal (including one confluent vessel) for small joints and two to three signals for large joints (including two confluent vessels);

Grade 2 = moderate confluent vessels (>grade 1 ) and less than $50 \%$ of normal area;

Grade 3 = marked vessels' signals in more than half the synovial area [2].

Linear Transducer (17 - 5 MHz) of Philips IU-22 machine was used for doppler assessment.

- ACR-EULAR 2010 criteria.

- Informed consent was taken from every patient in regional language and also in English.

- Clearance from the ethical committee was obtained.
- Statistical method: Two tailed fishers test.

\section{Result and Analysis}

In this study a total number of 106 patients of clinically suspected rheumatoid arthritis were studied as per selection criteria. All the patients were assessed by history taking and clinical examination. The laboratory investigations were recorded, including Hemogram, Sugar Creatinine, CRP, RF, and Anti-CCP.

Radiological examinations of hands were done by digital radiography and PDUS in a group of 53 patients, assessment of foot changes by PDUS and Digital Radiography were done in another similar group of 53 patients.

In digital radiographic assessment if the score is 1 or more, the patient is considered as positive for inflammatory arthritis by DR.

Similarly by PDUS, patient with a score of 1 or more is supposed as positive for inflammatory arthritis.

Final diagnosis by ACR EULAR-2010 criteria is done for all the patients.

The following results and observations obtained.

Age distribution (out of 106).

\begin{tabular}{ccc}
\hline Age group in yrs & No of patients & Percentage (\%) \\
\hline$<20$ y & 6 & 5.66 \\
$20-30$ & 8 & 7.54 \\
$30-40$ & 20 & 18.86 \\
$40-50$ & 32 & 30.18 \\
$50-60$ & 40 & 37.37 \\
Total & 106 & $100 \%$ \\
\hline
\end{tabular}

Sex distribution: 82 (77.36\%) patients were female, 24 (22.64\%) were male. Mean duration of disease was ten months.

Rheumatoid factor was positive in 65 (61.32\%) patients. Anti CCP was positive in 73 patients (68.86\%). CRP was positive in 64 (63.37\%) patients.

Digital radiography (DR) in hand.

\begin{tabular}{cccc}
\hline DR & $\begin{array}{c}\text { ACR-EULAR } \\
\text { positive }\end{array}$ & $\begin{array}{c}\text { ACR-EULAR } \\
\text { negative }\end{array}$ & Total \\
\hline Positive & 13 & 0 & 13 \\
Negative & 35 & 5 & 40 \\
Total & 48 & 5 & 53 \\
\hline
\end{tabular}

Specificity-100\%, sensitivity-27\%, positive predictive value-100\%, negative predictive value- $12.5 \%$.

Using two tailed fishers test, the p-value is 0.3174, i.e. nonsignificant. 
Power doppler ultrasound (PDUS) in hand.

\begin{tabular}{cccc}
\hline PDUS & $\begin{array}{c}\text { ACR-EULAR } \\
\text { positive }\end{array}$ & $\begin{array}{c}\text { ACR-EULAR } \\
\text { negative }\end{array}$ & Total \\
\hline Positive & 44 & 1 & 45 \\
Negative & 4 & 4 & 8 \\
Total & 48 & 5 & 53 \\
\hline
\end{tabular}

Specificity—80\%, sensitivity-91.66\%, positive predictive value— $97.77 \%$, negative predictive value- $50 \%$.

Digital radiography (DR) in feet.

\begin{tabular}{cccc}
\hline DR & $\begin{array}{c}\text { ACR-EULAR } \\
\text { positive }\end{array}$ & $\begin{array}{c}\text { ACR-EULAR } \\
\text { negative }\end{array}$ & Total \\
\hline Positive & 13 & 5 & 18 \\
Negative & 30 & 5 & 35 \\
Total & 43 & 10 & 53
\end{tabular}

Specificity—50\%, sensitivity-30\%, positive predictive value-72.22\%, negative predictive value- $14.28 \%$.

Using two tailed fishers test, the p-value is 0.2792 , i.e. non significant.

Power doppler ultrasound (PDUS) in feet.

\begin{tabular}{cccc}
\hline PDUS & $\begin{array}{c}\text { ACR-EULAR } \\
\text { positive }\end{array}$ & $\begin{array}{c}\text { ACR-EULAR } \\
\text { negative }\end{array}$ & Total \\
\hline Positive & 38 & 2 & 40 \\
Negative & 5 & 8 & 13 \\
Total & 43 & 10 & 53 \\
\hline
\end{tabular}

Specificity-80\%, sensitivity-88.37\%, positive predictive value-95\%, negative predictive value-61.53\%.

Using two tailed fishers test, the p-value is 0.0001 , i.e. significant.

Comparison of findings in hand and feet.

\begin{tabular}{cccccc}
\hline & Min & Max & $\begin{array}{c}\text { Mean } \\
{[95 \% \text { CI }]}\end{array}$ & SD & Total \\
\hline DR in hand & 0 & 6 & $\begin{array}{c}1.1 \\
{[0.72,1.48]}\end{array}$ & 1.914854421 & 53 \\
$\begin{array}{c}\text { DR in } \\
\text { feet }\end{array}$ & 0 & 5 & $\begin{array}{c}0.82 \\
{[0.54,1.10]}\end{array}$ & 1.395374755 & 53 \\
HP & 0 & 3 & $\begin{array}{c}0.62 \\
{[-0.00,1.24]}\end{array}$ & 3.139195033 & 53 \\
FP & 0 & 6 & $\begin{array}{c}1.34 \\
{[0.69,1.99]}\end{array}$ & 3.278719262 & 53 \\
\hline
\end{tabular}

HP: PDUS in hand; FP: PDUS in foot.

\section{Discussion}

The present study was carried over a period of 18 months on 106 patients, in the Department of Radiodiagnosis and
Rheumatology Unit at Kolkata Medical College and Hospital, Kolkata, India.

The patients with polyarthritis and morning stiffness for more than one hour with disease duration of less than two years were referred with clinical diagnosis of rheumatoid arthritis.

Digital X-ray of hands and feet were taken and Ultrasound of small joints of hand and foot were done in our department. The thorough history and clinical examinations were done and all the relevant biochemical values were checked. Finally patients were classified according to ACR-EULAR 2010 CRITERIA were diagnosed as suffering from early rheumatoid arthritis if they score 6 or more.

Of the total 106 patients, a group of 53 patients was examined for hand changes and another similar group of 53 patients was examined for foot changes. Mean duration of disease was ten months.

In hand Digital Radiography (DR) were positive in 13 patients of which all the 13 patients were diagnosed as suffering from early RA by ACR EULAR 2010,DR was negative in 40 patients of which 35 were diagnosed as R.A. patient and 5 patients were not suffering from R.A.

The p-value was 0.3174 i.e., not significant.

PDUS were positive showing synovial vascularity in 45 patients of which 44 were confirmed as suffering from early RA by ACR-EULAR 2010.

PDUS were negative in 8 cases of which 4 patients were confirmed RA patients by ACR EULAR-2010 criteria.

The p-value was 0.0011 i.e. significant.

In feet, DR positive patients were 18 in number of which 13 cases were diagnosed ACR-EULAR 2010 as suffering from early RA, DR negative case were 35 in number and of which 30 were diagnosed by ACR-EULAR 2010 as suffering from early RA.

p-value was 0.2792 i.e. not significant

PDUS positive patients were 40 in number and 38 of them were confirmed by ACR-EULAR 2010 to be suffering from early RA, PDUS were negative to show synovitis in 13 patients, of which 8 patients were truly diagnosed as early RA patients.

p-value is 0.0001 i.e. significant.

So the comparative study reveals that synovial vascularity as demonstrated by PDUS is much more effective in diagnosing early rheumatoid arthritis, both in hand and in feet than digital radiograph.

The sensitivity of PDUS in foot is more than that of hand.

According to Sutton 7th edition [3] erosions appear earlier and are more often seen in the feet, $90 \%$ of which will eventually be affected, than in the hands (75\%), and most often at the fifth metacarpophalangeal joint. Ero- 
sions affect typically the lateral side of the fifth metatarsal but the medial side of the others.

Devauchelle Pensec V. et al. [4] studied patients with arthritis of less than one year duration was evaluated in a multicenter study and followed for $30+/-11$ months. An observer blinded to patient data read all 149 hand and foot radiographs done at study inclusion, using item 7 of the 1987 American College of Rheumatology (ACR) criteria [5] for RA and Sharp's method [6] to score erosions and joint space narrowing.

The kappa coefficient for the 1987 ACR item 7 was 0.52 for bony decalcification and 0.87 for erosions. Intra and inter observer correlation coefficients for Sharp's scores ranged from 0.90 to 0.98 . Erosions at the feet were significantly associated with RA. The item 7 erosion component at the feet was more specific than the full item 7 (97.5\% vs. 94\%; p = 0.01). Sharp's erosion score at the feet was not better than the erosion component of item 7 (sensitivity 18\%; specificity 97.5\%). Combined use of radiographs of the hands and feet improved the diagnostic performance of the item 7 erosion component; (sensitivity and specificity of item 7 erosions at the hands combined with the feet were $32.5 \%$ and $94.5 \%$, respectively).

It was concluded that the "erosion" criterion at the feet had the best diagnostic performance and was significantly associated with a diagnosis of RA. Combining hand and foot radiographs improved diagnostic performance [4].

In the present study, X-ray of hands and feet by Digital Radiography was not a very efficient tool for diagnosis of early Rheumatoid arthritis.

Power Doppler ultrasound played a very good role in evaluation of vascularity of synovium in both hands and feet of patients suffering from early Rheumatoid Arthritis.

In comparison to hands, synovial vascularity as assessed by PDUS was more common in feet in those patients.

Synovial vascularity as detected by PDUS has a good correlation with ACR EULAR-2010 criteria for early RA patients.

The correlation is more significant in feet than in hands.

Thus, we may conclude that Power Doppler Ultrasonography of synovium of small joints of hands and feet is a very useful tool in assessing both inflammatory and destructive changes and help the clinician to start the appropriate medication at the earliest stage of the disease, in a developing country like India, where the cost of investigations is a significant factor for most patients. PDUS of feet may yield earlier and better findings than hands, which is conventionally used in patients suffering from early rheumatoid arthritis.

\section{Limitations}

Larger study with more number of cases will be more helpful in assessing the exact roles of Power Doppler Ultrasonography in patients suffering from early rheumatoid arthritis.

\section{Disclosure}

Authors declare that there is no conflict of interests regarding the publication of this article. Authors of the manuscript do not have a direct financial relation with the commercial identities mentioned in this paper that might lead to a conflict of interest for any of the authors.

\section{REFERENCES}

[1] D. Aletaha, T. Neogi, A. J. Silman, et al., "Rheumatoid Arthritis Classification Criteria: An American College of Rheumatology/European League against Rheumatism Collaborative Initiative,” Annals of the Rheumatic Diseases, Vol. 69, No. 9, 2010, pp. 1580-1588. http://dx.doi.org/10.1136/ard.2010.138461

[2] M. Dougados, et al., "The Ability of Synovitis to Predict Structural Damage in Rheumatoid Arthritis: A Comparative Study between Clinical Examination and Ultrasound," Annals of the Rheumatic Diseases, Vol. 72, No. 5, 2013, pp. 665-671. http://dx.doi.org/10.1136/annrheumdis-2012-201469

[3] “Text Book of Radiology and Imaging,” 7th Edition, Vol. 2, David Sutton, p. 1201.

[4] V. Devauchelle Pensec, A. Saraux, J. M. Berthelot, S. Alapetite, et al., "Ability of Foot Radiographs to Predict Rheumatoid Arthritis in Patients with Early Arthritis," Journal of Rheumatology, Vol. 31, No. 1, 2004, pp. 6670.

[5] F. C. Arnett, S. M. Edworthy, D. A. Bloch, et al., "The American Rheumatism Association 1987 Revised Criteria for the Classification of Rheumatoid Arthritis," Arthritis \& Rheumatism, Vol. 31, No. 3, 1988, pp. 315-324. http://dx.doi.org/10.1002/art.1780310302

[6] D. Van der Heijde, "How to Read Radiographs According to the Sharp/van der Heijde Method. Prediction of Radiological Outcome in Early Rheumatoid Arthritis in Clinical Practice,” Journal of Rheumatology, Vol. 26, 1999, pp. 743-745. 